\title{
Posterior Floating Laminotomy as a New Decompression Technique for Posterior Cervical Spinal Fusion Surgery
}

\author{
Hong Kyung Shin, Jin Hoon Park \\ Department of Neurological Surgery, Asan Medical Center, University of Ulsan College of Medicine, Seoul, Korea
}

Objective : In the cervical spine, many surgical procedures have been developed to achieve optimal results for various disorders, including degenerative diseases, traumatic injury, and tumor. In this study, we report our experience and follow-up results with a new surgical technique for cervical spine entitled posterior floating laminotomy (PFL) in comparison with conventional laminectomy and fusion (LF).

Methods : Data for 85 patients who underwent conventional LF ( $n=66)$ or PFL $(n=19)$ for cervical spine disorders between 2012 and 2019 were analyzed. Radiological parameters, including cervical lordosis (CL), T1 slope (T1S), segmental lordosis (SL), and C2-7 sagittal vertical axis (SVA), were measured with lateral spine X-rays. Functional outcomes, comprising the modified Japanese Orthopaedic Association (mJOA), neck disability index (NDI), and visual analog scale (VAS) scores, were also measured. For the patients who underwent PFL, postoperative magnetic resonance image (MRI) was performed in a month after the surgery, and the degree of decompression was evaluated at the T2-weighted axial image, and postoperative computed tomography (CT) was conducted immediately and 1 year after the operation to evaluate the gutter fusion.

Results : There was no difference in CL, T1S, SL, and C2-7 SVA between the groups but there was a difference in the preoperative and postoperative $S L$ angles. The mean difference in the preoperative $S L$ angle compared with that at the last follow-up was $-0.3^{\circ}$ after conventional LF and $4.7^{\circ}$ after PFL $(p=0.04)$, respectively. $\mathrm{mJOA}, \mathrm{NDI}$, and VAS scores showed significant improvements $(p<0.05)$ during follow-up in both groups. In the PFL group, postoperative MRI showed sufficient decompression and postoperative CT revealed gutter fusion at 1 year after the operation.

Conclusion : PFL is a safe surgical method which can preserve postoperative $C L$ and achieve good clinical outcomes.

Key Words : Cerivcal vertebrae · Pedicle screw · Spinal fusion · Lateral mass screw · Posterior floating laminotomy.

\section{INTRODUCTION}

The primary goal of spinal surgery is to attain sufficient neural recovery from the damaged spinal cord and roots. Sev- eral methods have been developed to achieve optimal results for various disorders in the cervical spine, including degenerative diseases, traumatic injuries, and tumors. There are two main surgical approaches employed in the cervical spine : the

- Received : October 23, 2020 •Revised : December 23, 2020 •Accepted : Febraury 23, 2021

- Address for reprints : Jin Hoon Park

Department of Neurological Surgery, Asan Medical Center, University of Ulsan College of Medicine, 88 Olympic-ro 43-gil, Songpa-gu, Seoul 05505, Korea Tel : +82-2-3010-3550, Fax : +82-2-476-6738, E-mail : jhpark@amc.seoul.kr, ORCID : https://orcid.org/0000-0002-0903-3146 
anterior approach and the posterior approach. Anterior cervical surgery includes anterior cervical discectomy and fusion, and posterior cervical surgery includes laminectomy alone, laminoplasty, and laminectomy and fusion (LF).

The anterior approach surgery has an advantage due to its direct decompression of compromised structures. However, the rates of dysphagia and recurrent laryngeal nerve palsy are relatively high compared with the rates obtained with the posterior approach ${ }^{13)}$. Additionally, the degeneration of adjacent levels can occur with the anterior procedure ${ }^{2)}$. Posterior approaches, including laminectomy alone, laminoplasty, and LF, are usually conducted in cases of multilevel cord compression. Shifting the spinal cord posteriorly induces indirect decompression. However, laminectomy alone is generally avoided due to the risk of delayed postlaminectomy kyphosis ${ }^{11)}$. Laminoplasty is performed as an alternative to laminectomy, achieving sufficient decompression and mechanical solidity ${ }^{3,4)}$. LF provides better long-term stability than laminectomy alone by inducing arthrodesis and has a wider surgical indication than laminoplasty as it facilitates lordotic change from kyphosis $^{1)}$. However, complications after posterior surgery, such as axial pain, C5 palsy, postoperative kyphosis, and pseudomembrane formation are not uncommon ${ }^{7,12)}$.

The appropriate surgical method for cervical disorders has been controversial for many years. In this study, we present an innovative surgical method for cervical spine disorders entitled posterior floating laminotomy (PFL), which overcomes the drawbacks of LF while retaining the strengths of laminoplasty.

\section{MATERIALS AND METHODS}

\section{Patient enrollment}

This study was performed under an Institutional Review Board-approved protocol in compliance with regulations set by Gangneung Asan Hospital (IRB No. 2019-11-006), and no patient consent was required as no identifying material has been included. We retrospectively reviewed the medical records of patients who underwent cervical spinal surgery between 2012 and 2019. A total of 85 patients were enrolled. Conventional LF was performed on 66 patients from 2012 to 2019, while PFL was consecutively performed on 19 patients from 2016 to 2019. From 2016, the surgical method was deter- mined on a case by case basis. Surgery indications were trauma, cervical spondylotic myelopathy, ossification of posterior longitudinal ligament, foraminal stenosis, and tumor. Surgical indications were not different between the groups and detailed classification are described in Table 1. Exclusion criteria were patients with infection, corpectomy, and fusion including the $\mathrm{C} 1$ or below the $\mathrm{T} 2$ level.

\section{Surgical methods}

A cervical pedicle screw (CPS) and a lateral mass screw (LMS) were used for both the conventional LF group and the PFL group. When ball-tip probing, feedback suggestive of malpositioning of the screw led to abandonment of the CPS procedure and conversion to LMS insertion.

In the conventional LF group, after confirmation of the screw position by anterior-posterior and lateral radiographs, laminectomy was performed using a high-speed drill with a small round burr $(1.8 \mathrm{~mm})$. Additionally, decorticated local

Table 1. Baseline characteristics of patients in conventional LF and PFL groups

\begin{tabular}{|c|c|c|c|}
\hline & $\begin{array}{c}\text { Conventional } \\
\text { LF }\end{array}$ & PFL & $p$-value \\
\hline Number of patients & 66 & 19 & \\
\hline Age at surgery (years) & $66.1 \pm 11.9$ & $64.1 \pm 13.4$ & 0.53 \\
\hline Sex & & & 0.31 \\
\hline Male & $56(84.8)$ & $14(73.7)$ & \\
\hline Female & $10(15.2)$ & $5(26.3)$ & \\
\hline DM & $14(21.2)$ & $6(31.6)$ & 0.37 \\
\hline HTN & $30(45.5)$ & $7(36.8)$ & 0.69 \\
\hline Smoking & $26(39.4)$ & $8(42.1)$ & 1.00 \\
\hline $\mathrm{BMI}\left(\mathrm{kg} / \mathrm{m}^{2}\right)$ & $24.1 \pm 3.2$ & $24.3 \pm 4.7$ & 0.83 \\
\hline Osteoporosis & $3(4.5)$ & $1(5.3)$ & 1.00 \\
\hline Previous cervical operation & $6(9.1)$ & $4(21.1)$ & 0.22 \\
\hline Classification & & & 0.24 \\
\hline Trauma & $36(54.5)$ & $6(31.6)$ & \\
\hline CSM & $18(27.3)$ & $9(47.4)$ & \\
\hline OPLL & $7(10.6)$ & $3(15.8)$ & \\
\hline Foraminal stenosis & $3(4.5)$ & $0(0.0)$ & \\
\hline Tumor & $2(3.0)$ & $1(5.3)$ & \\
\hline
\end{tabular}

Values are presented as mean \pm standard deviation or number (\%). LF : laminectomy and fusion, PFL : posterior floating laminotomy, DM : diabetes mellitus, HTN : hypertension, BMI : body mass index, CSM : cervical spondylotic myelopathy, OPLL : ossification of posterior longitudinal ligament 
bone (i.e., spinous process and lamina bone chips) mixed with allobone was applied over the duraform, which covered the spinal cord, to promote segmental fusion and was used for posterolateral fusion. In the PFL group, laminotomy was per-

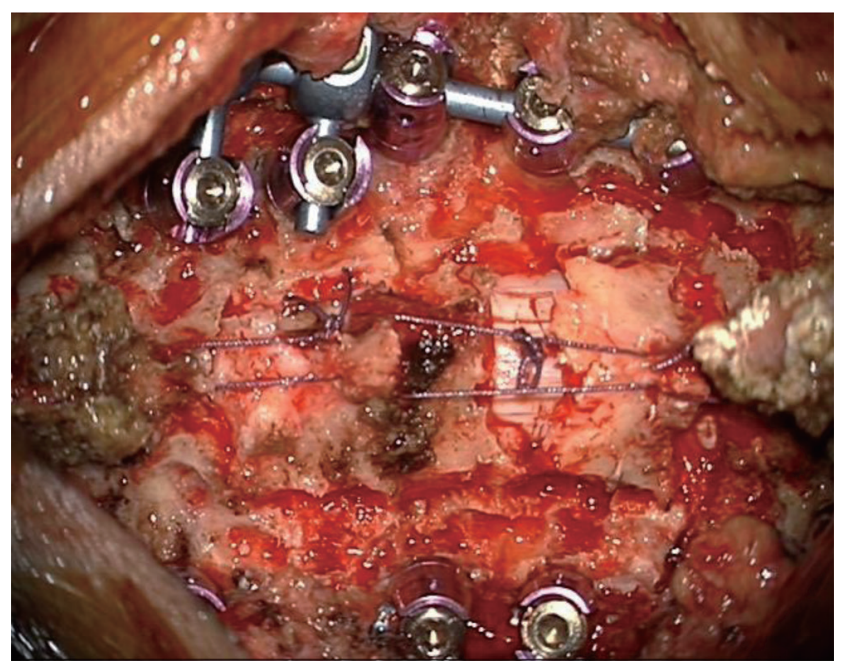

Fig. 1. Posterior bony structures are restored on the spinal cord and linked to the adjacent paraspinal muscles and tendons in order to protect the cord and fill the empty space in the posterior floating laminotomy group. formed using a high-speed drill with a small round burr to make gutter. Next, only the lamina without ligamentum flavum was disconnected en bloc and shifted to posteriorly with Allis forceps while some proportion of ligamentum flavum was preserved, since the isolated lamina is unstable and difficult to reattach to the original sites. If the inner layer of the lamina compressed the spinal cord even after posterior shifting, additional internal decompression through removal of inner wall of lamina, central part of ligamentum flavum, or epidural fat was performed. After shifting posteriorly, floating lamina was adhered to the paraspinal muscles, tendons, and upper and lower spinous processes with threads (Fig. 1). Lastly, decorticated bone chips were applied at the gutter to make bone fusion for long-term stability. Schematic images of the surgery are illustrated (Fig. 2).

\section{Outcome evaluation}

A comprehensive review of medical records was performed to gather data regarding the baseline characteristics, radiological parameters, and functional outcomes. The baseline characteristics of the patients, including age, sex, diabetes mellitus (DM), hypertension (HTN), smoking, body mass in-

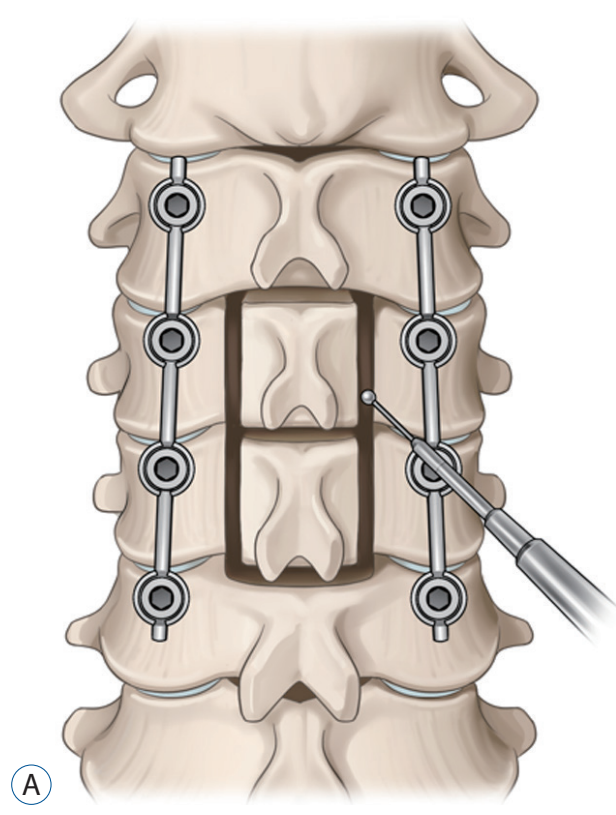

(B)
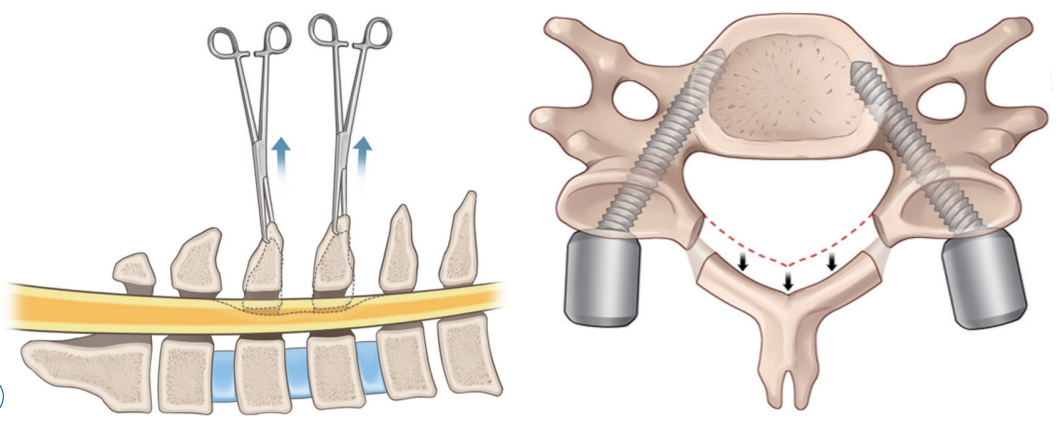

(C)

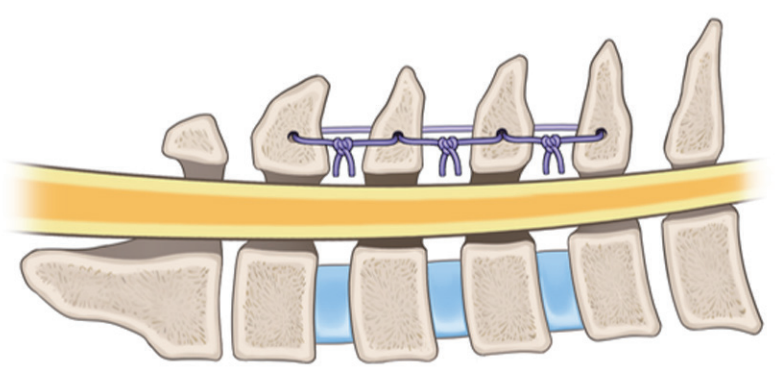

Fig. 2. Schematic images of the posterior floating laminotomy surgery. A : Laminotomy was performed using a high-speed drill with a small round burr to make gutter. B : Only the lamina without ligamentum flavum was disconnected en bloc and shifted to posteriorly with Allis forceps while some proportion of ligamentum flavum was preserved, since the isolated lamina is unstable and difficult to reattach to the original sites. If the inner layer of the lamina compressed the spinal cord even after posterior shifting, additional internal decompression through removal of inner wall of lamina, central part of ligamentum flavum, or epidural fat was performed. C : Posteriorly shifted floating lamina was adhered to the paraspinal muscles, tendons, and upper and lower spinous processes with threads. 
dex (BMI), osteoporosis, history of previous cervical operation, and classification, were recorded.

The radiological parameters were analyzed with lateral spine X-rays before surgery, immediately after surgery, and at the last follow-up. We measured cervical lordosis (CL), the T1 slope (T1S), segmental lordosis (SL), and the C2-7 sagittal vertical axis (SVA) on lateral spine X-rays. CL, defined as the C2-7 Cobb angle, was evaluated based on the angle between the lower endplate of the C2 vertebra and the lower endplate of the C7 vertebra. T1S was evaluated based on the angle be-

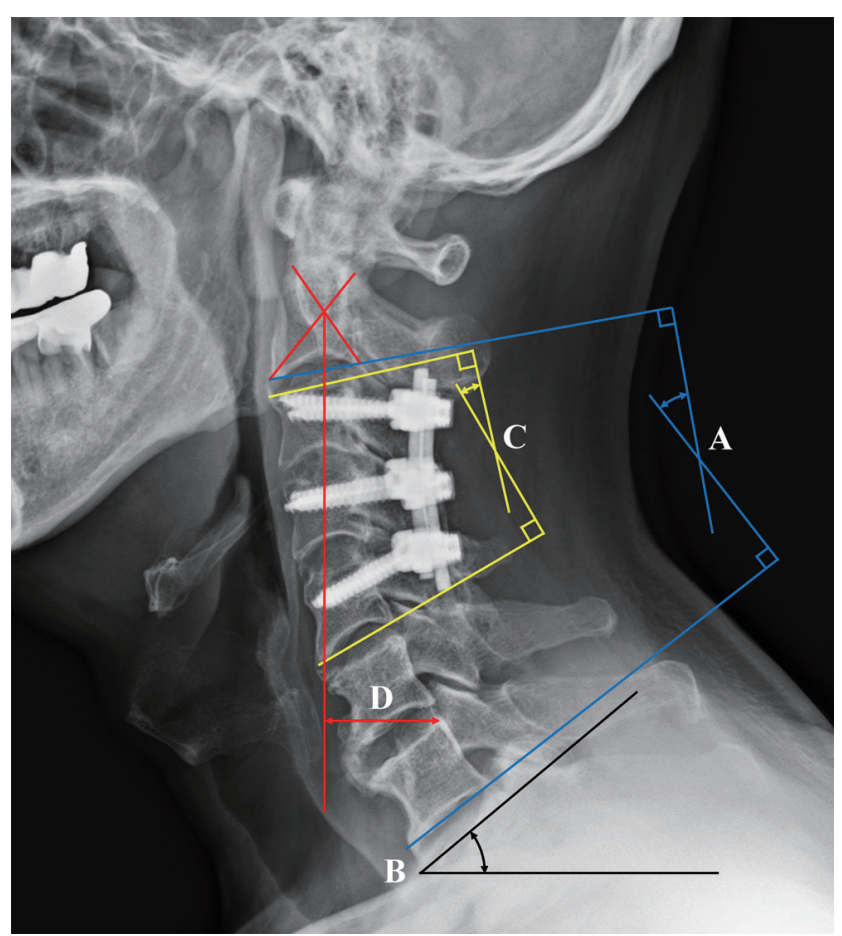

Fig. 3. Radiological parameters are shown. A : Cervical lordosis. B : T1 slope. C : Segmental lordosis. D : C2-7 sagittal vertical axis.

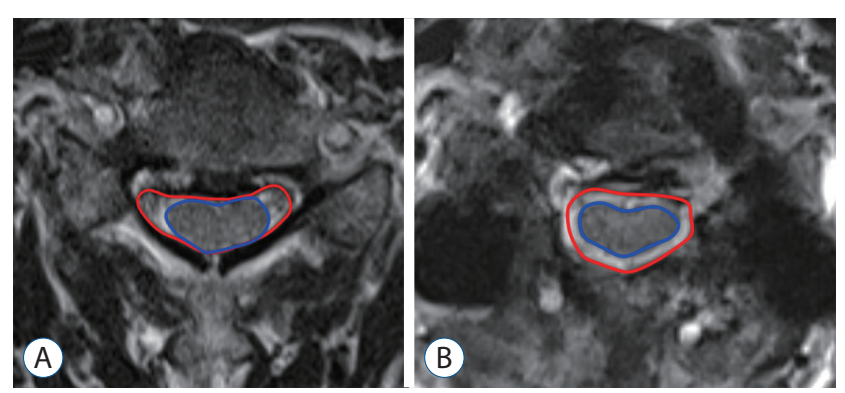

Fig. 4. From the T2-weighted axial magnetic resonance image, the area of the cord (marked as blue line) and the spinal canal (marked as red line) were measured from $C 3$ to $C 7$, before $(A)$ and after $(B)$ the posterior floating laminotomy surgery. tween the upper endplate of T1 and the horizontal line. SL was evaluated from the angle between the upper endplate of the upper instrumented vertebra and the lower endplate of the lower instrumented vertebra. C2-7 SVA was measured as the

Table 2. Radiological parameters for patients in conventional LF and PFL groups

\begin{tabular}{|c|c|c|c|}
\hline & $\begin{array}{c}\text { Conventional } \\
\text { LF }\end{array}$ & PFL & $p$-value \\
\hline \multicolumn{4}{|c|}{ Preoperative $\left(^{\circ}\right)$} \\
\hline $\mathrm{CL}$ & $12.9 \pm 12.9$ & $11.4 \pm 13.4$ & 0.69 \\
\hline T1S & $27.8 \pm 8.8$ & $28.0 \pm 9.0$ & 0.91 \\
\hline SL & $9.2 \pm 10.2$ & $7.6 \pm 9.6$ & 0.57 \\
\hline C2-7 SVA & $26.2 \pm 12.3$ & $21.4 \pm 11.8$ & 0.17 \\
\hline \multicolumn{4}{|c|}{ Immediate postoperative $\left(^{\circ}\right)$} \\
\hline $\mathrm{CL}$ & $15.8 \pm 9.4$ & $14.9 \pm 10.1$ & 0.75 \\
\hline $\mathrm{T} 1 \mathrm{~S}$ & $29.4 \pm 9.0$ & $32.3 \pm 7.8$ & 0.24 \\
\hline SL & $11.2 \pm 8.4$ & $12.7 \pm 6.7$ & 0.51 \\
\hline C2-7 SVA & $27.4 \pm 12.1$ & $27.0 \pm 15.3$ & 0.89 \\
\hline \multicolumn{4}{|c|}{ Last follow-up $\left(^{\circ}\right)$} \\
\hline $\mathrm{CL}$ & $13.4 \pm 9.3$ & $12.7 \pm 10.7$ & 0.78 \\
\hline T1S & $27.9 \pm 7.5$ & $28.6 \pm 10.3$ & 0.77 \\
\hline SL & $8.9 \pm 7.7$ & $11.5 \pm 8.0$ & 0.25 \\
\hline C2-7 SVA & $26.8 \pm 13.5$ & $28.9 \pm 14.3$ & 0.60 \\
\hline \multicolumn{4}{|c|}{$\begin{array}{l}\Delta \text { Last follow-up - immediate } \\
\text { postoperative }\left(^{\circ}\right)\end{array}$} \\
\hline $\mathrm{CL}$ & $-2.3 \pm 8.1$ & $-2.1 \pm 9.1$ & 0.95 \\
\hline $\mathrm{T} 1 \mathrm{~S}$ & $-1.4 \pm 7.6$ & $-3.7 \pm 10.5$ & 0.34 \\
\hline SL & $-2.3 \pm 5.8$ & $-1.2 \pm 5.9$ & 0.51 \\
\hline C2-7 SVA & $-0.6 \pm 10.8$ & $1.8 \pm 17.2$ & 0.48 \\
\hline \multicolumn{4}{|c|}{$\begin{array}{l}\Delta \text { Immediate postoperative - } \\
\text { preoperative }\left(^{\circ}\right)\end{array}$} \\
\hline $\mathrm{CL}$ & $2.9 \pm 12.7$ & $3.5 \pm 9.9$ & 0.86 \\
\hline T1S & $1.6 \pm 9.6$ & $4.3 \pm 11.3$ & 0.35 \\
\hline$S L$ & $2.0 \pm 10.5$ & $5.1 \pm 9.4$ & 0.29 \\
\hline C2-7 SVA & $1.2 \pm 15.1$ & $5.5 \pm 15.6$ & 0.32 \\
\hline \multicolumn{4}{|c|}{$\begin{array}{l}\Delta \text { Last follow-up - } \\
\text { preoperative }\left(^{\circ}\right)\end{array}$} \\
\hline $\mathrm{CL}$ & $0.6 \pm 11.4$ & $1.3 \pm 11.9$ & 0.82 \\
\hline T1S & $0.1 \pm 8.9$ & $0.5 \pm 9.2$ & 0.87 \\
\hline$S L$ & $-0.3 \pm 8.5$ & $4.7 \pm 7.2$ & 0.04 \\
\hline C2-7 SVA & $0.6 \pm 14.4$ & $7.4 \pm 16.1$ & 0.11 \\
\hline
\end{tabular}

Values are presented as mean \pm standard deviation. LF : laminectomy and fusion, PFL : posterior floating laminotomy, CL : cervical Lordosis, T1S : T1 slope, SL : segmental lordosis, SVA : sagittal vertical axis 
distance between a plumb line from the center of the $\mathrm{C} 2$ body to the superior posterior endplate of C7. These measurement methods are presented in Fig. 3.

In addition to radiological parameters, functional outcomes were obtained before surgery, immediately after surgery, and at the last follow-up. Functional outcomes consisted of modified Japanese Orthopaedic Association (mJOA), neck disability index (NDI), and visual analog scale (VAS) scores. The mJOA score is a quantitative scale that evaluates the severity of myelopathy. The scale is representative of four components : 1) motor function in the arms, 2) motor function in the legs, 3) sensation, and 4) bladder function. Scores are estimated from 0 to 17, with a lower score correlating with higher dis- ability $^{10)}$. The NDI score is a clinical assessment of disability, specific to the cervical spine, which has a high degree of reliability and internal consistency ${ }^{28}$. The scores are estimated from 0 to 50, with a higher score correlating with higher disability. Pain severity was measured with the VAS score, with VAS for the neck (VAS-N) and the arm (VAS-A). The VAS score was measured from 0 to 10 , with 0 representing no pain and 10 representing the worst pain ${ }^{15}$.

We performed the postoperative magnetic resonance image (MRI) in 1 month after the PFL surgery to evaluate the degree of decompression. From the T2-weighted axial images, the area of the cord and the spinal canal were measured at each pedicle level from C3 to C7, before and after the surgery (Fig. 4).
$\mathrm{CL}$



$S L$

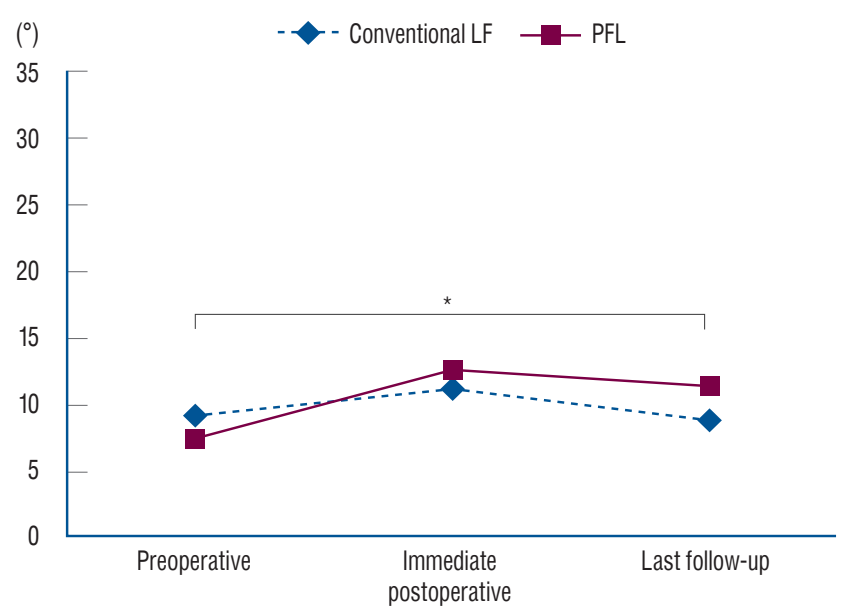

T1S

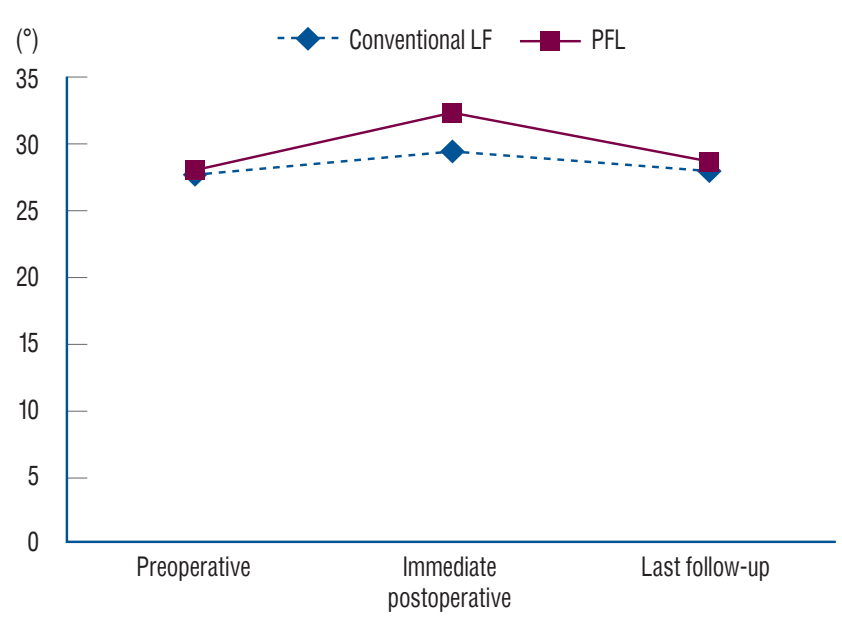

C2-7 SVA

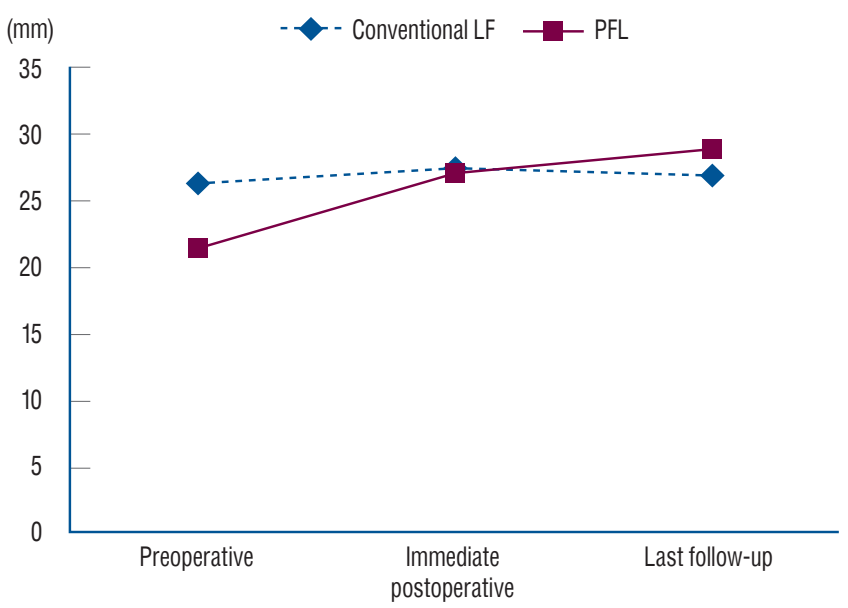

Fig. 5. Radiological parameters, including $C L, T 1 S, S L$, and C2-7 SVA, were not different between the groups. However, there was a difference in SL between the preoperative time and the last follow-up (marked with a star). The difference was $-0.3^{\circ}$ in the conventional $\mathrm{LF}$ group and $4.7^{\circ}$ in the PFL group $(p=0.04)$. CL : cervical lordosis, LF : laminectomy and fusion, PFL : posterior floating laminotomy, T1S : T1 slope, SL : segmental lordosis, SVA : sagittal vertical axis. 
To discriminate the subarachnoid space from the ligamentous or osseous structures, we set the borderline of the spinal canal area (i.e., the subarachnoid space) along the inner margin of low signal intensity ${ }^{22,25)}$. The measurements were viewed on Surgimap software version 2.3.1 (Nemaris Inc., New York, NY, USA). All parameters were measured independently by two observers, and the average value of each parameter at each level was taken for independent calculations.

Postoperative computed tomography (CT) images were obtained immediately and 1 year after the operation to evaluate fusion status. Fusion was defined as definite bony bridging on axial CT images. Perioperative data such as the number of levels fused, screw fixation method, estimated blood loss (EBL), length of hospital stay (LOS), operation time, non-pathologic fever defined as a fever without an infection ${ }^{26)}$, and perioperative complications were also collected. Complications were defined as any subsequent event after the operation requiring further treatment.

\section{Statistical analysis}

Quantitative data were presented as the mean \pm standard deviation unless otherwise indicated. Independent T-tests were used to assess continuous variables. Chi-square test and Fisher's exact test were used for categorical variables. Significant differences in functional outcomes among the three different time points were assessed using one-way repeated measures analysis of variance (ANOVA). We performed a Bonferroni correction on one-way repeated measures ANOVA and the variables were considered statistically significant with $p<0.017$. For all the other analyses, the results were considered to show statistical significance when $p<0.05$. All statistical tests were two-sided. We used R version 3.6.1 (R Foundation for Statistical Computing, Vienna, Austria) for all statistical analyses.

\section{RESULTS}

Of the 85 included patients, 66 patients received conventional LF and 19 received PFL. There was no difference between the groups regarding the age $(p=0.53)$, sex $(p=0.31)$, DM $(p=0.37)$, HTN $(p=0.69)$, smoking $(p=1.00)$, BMI $(p=0.83)$, osteoporosis $(p=1.00)$, history of previous cervical operation $(p=0.22)$, and classification $(p=0.24)$. These baseline characteristics are summarized in Table 1 . After the operation, all degenerative patients demonstrated neurological improvement and none of the trauma patients with spinal cord injury experienced aggravation of their neurological function in either group, except one case of restenosis in PFL group due to the postoperative cervical trauma.

Radiological parameters, including CL, T1S, SL, and C2-7 SVA, were not different between the groups, but a difference was observed in the SL angle preoperatively and at the last follow-up. SL difference between the preoperative time and the last follow-up was $-0.3^{\circ}$ in the conventional LF group and $4.7^{\circ}$

Table 3. Functional outcomes for patients in conventional LF and PFL groups

\begin{tabular}{|c|c|c|c|c|}
\hline & Preoperative & Postoperative 6 months & Postoperative 12 months & Overall $p$-value \\
\hline \multicolumn{5}{|c|}{ Conventional LF } \\
\hline $\mathrm{mJOA}$ & $10.9 \pm 3.2$ & $13.9 \pm 2.4^{*}$ & $14.6 \pm 2.6^{\dagger}$ & $<0.01$ \\
\hline $\mathrm{NDI}$ & $31.3 \pm 9.7$ & $17.2 \pm 7.0^{*}$ & $12.2 \pm 6.8^{\dagger}$ & $<0.01$ \\
\hline VAS-N & $7.2 \pm 1.6$ & $2.0 \pm 1.6^{*}$ & $1.5 \pm 1.2^{\dagger}$ & $<0.01$ \\
\hline VAS-A & $6.7 \pm 2.0$ & $2.1 \pm 1.8^{*}$ & $1.6 \pm 1.0^{\dagger}$ & $<0.01$ \\
\hline \multicolumn{5}{|l|}{ PFL } \\
\hline $\mathrm{mJOA}$ & $11.7 \pm 4.3$ & $15.5 \pm 2.0$ & $16.8 \pm 1.6^{\dagger}$ & $<0.01$ \\
\hline NDI & $21.2 \pm 10.0$ & $10.0 \pm 5.8$ & $9.0 \pm 6.3$ & 0.01 \\
\hline VAS-N & $5.7 \pm 2.2$ & $3.5 \pm 1.1^{*}$ & $2.2 \pm 1.1^{\dagger}$ & $<0.01$ \\
\hline VAS-A & $6.1 \pm 2.4$ & $4.1 \pm 2.2^{*}$ & $2.5 \pm 2.0^{\dagger}$ & $<0.01$ \\
\hline
\end{tabular}

Values are presented as mean \pm standard deviation. * Statistically significant difference (with Bonferroni correction, $p<0.017$ [0.05/3=0.017]) between the preoperative and the postoperative 6 months. ${ }^{\dagger}$ Statistically significant difference (with Bonferroni correction, $p<0.017[0.05 / 3=0.017]$ ) between the preoperative and the postoperative 12 months. LF : laminectomy and fusion, PFL : posterior floating laminotomy, mJOA : modified Japanese Orthopaedic Association, NDI : neck disability index, VAS-A : visual analog scale for the arm, VAS-N : visual analog scale for the neck 
in the PFL group ( $p=0.04$ ) (Table 2 and Fig. 5). Functional outcomes, consisting of mJOA, NDI, VAS-N, and VAS-A scores, were measured at the preoperative time, postoperative 6 months, and postoperative 12 months and showed significant differences $(p<0.05)$ in each group (Table 3 and Fig. 6).

The area of the cord and the spinal canal from C3 to C7 which was measured at the T2-weighted axial MRI are presented in Table 4 and Fig. 7. There were statistically significant differences in the area of the spinal canal from C3 to C7, between before and after the surgery. Follow-up CT 1 year after the operation revealed the formation of bony continuity at the gutter between the lateral mass and the lamina, whereas im- mediate postoperative CT showed a gap between the lateral mass and the lamina (Figs. 8 and 9). Although it was difficult to specify when the bone fusion occurred, it was possible to identify the fusion status with a CT scan.

Perioperative outcomes are presented in Table 5. The numbers of levels fused after conventional LF and PFL were 2.8 and $3.0(p=0.48)$, respectively. Screw fixation methods were not different between the groups $(p=0.42)$. Although the total operation times for the conventional LF group and the PFL group were different (conventional LF : 169.6 vs. PFL : 223.7 minutes, $p=0.01$ ), the operation times per fusion level were not (conventional LF : 72.3 vs. PFL : 90.2 minutes, $p=0.08$ ). EBL
$\mathrm{MJOA}$

Preoperative $\square$ Postoperative 6 months $\square$ Postoperative 12 months



VAS-N

Preoperative $\square$ Postoperative 6 months $\square$ Postoperative 12 months

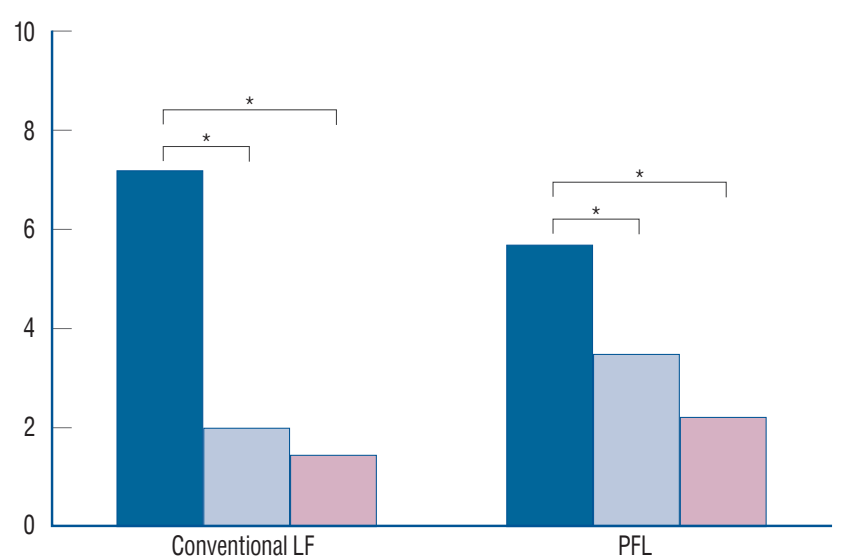

NDI

Preoperative $\square$ Postoperative 6 months $\square$ Postoperative 12 months

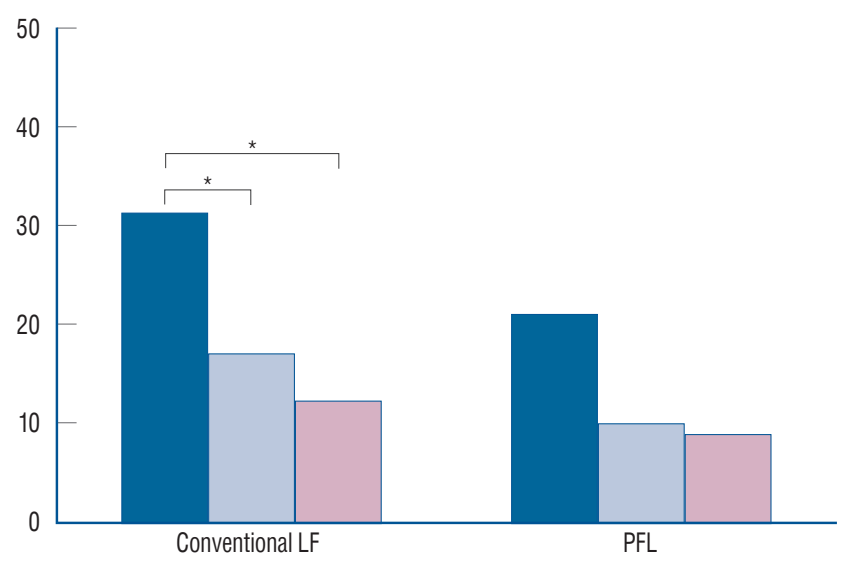

VAS-A

Preoperative $\square$ Postoperative 6 months $\square$ Postoperative 12 months

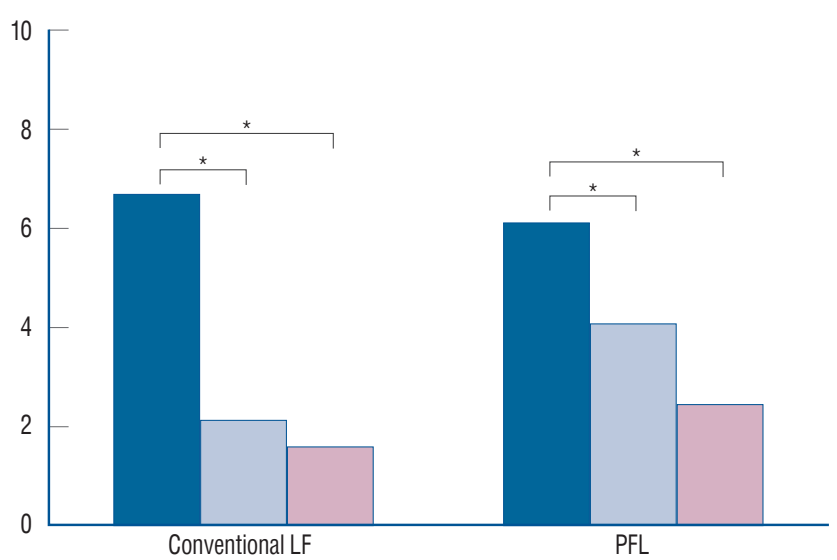

Fig. 6. Functional outcomes, including mJOA, NDI, VAS-N, and VAS-A scores, at three time points (preoperative, postoperative 6 months, and postoperative 12 months) were significantly different $(p<0.05)$ in both groups. There were also significant differences between the preoperative score and the scores 6 and 12 months postoperatively with Bonferroni correction (marked with stars). mJOA : modified Japanese Orthopaedic Association, LF : laminectomy and fusion, PFL : posterior floating laminotomy, NDI : neck disability index, VAS-N : visual analog scale for the neck, VAS-A : visual analog scale for the arm. 
$(p=0.28)$, LOS $(p=0.99)$, and non-pathologic fever $(p=0.34)$ were not different between the groups. Wound infection occurred in five patients in the conventional LF group and did not occur in the PFL group ( $p=0.58)$. Seroma, identified in

Table 4. Area of the cord and the spinal canal from C3 to C7 at axial MRI, before and after the PFL surgery

\begin{tabular}{lccc}
\hline Area $\left(\mathrm{mm}^{2}\right)$ & Before the surgery & After the surgery & $p$-value \\
\hline C3 & & & \\
Cord & $68.1(7.7)$ & $70.3(7.2)$ & 0.69 \\
Canal & $139.2(26.3)$ & $183.9(10.5)$ & $0.02^{*}$ \\
C4 & & \\
Cord & $89.1(19.6)$ & $89.7(19.1)$ & 0.97 \\
Canal & $120.5(33.5)$ & $171.9(20.3)$ & $0.04^{*}$ \\
C5 & & & \\
Cord & $76.6(18.0)$ & $84.1(1.8)$ & 0.61 \\
Canal & $93.6(17.5)$ & $165.0(14.1)$ & $0.04^{*}$ \\
C6 & & & \\
Cord & $81.4(3.9)$ & $79.7(4.3)$ & 0.57 \\
Canal & $119.4(23.7)$ & $165.7(23.2)$ & $0.03^{*}$ \\
C7 & & & \\
Cord & $63.5(10.6)$ & $68.3(10.0)$ & 0.52 \\
Canal & $133.8(20.3)$ & $164.7(8.4)$ & $0.03^{*}$ \\
\hline
\end{tabular}

Values are presented as number (\%). *Statistically significant difference $(p<0.05)$ between before and after the PFL surgery. MRI : magnetic resonance image, PFL : posterior floating laminotomy
MRIs, occurred in six patients in the conventional LF group and in one patient in the PFL group ( $p=1.00)$ (Fig. 10). C5 palsy occurred in one patient in each group $(p=0.39)$. Screw fracture occurred in one patient in the conventional LF group and did not occur in the PFL group $(p=1.00)$. Restenosis due to postoperative cervical trauma occurred in one patient in the PFL group and did not occur in the conventional LF group ( $p=0.22$ ). The mean durations of postoperative follow-up were different between the groups (conventional LF : 35.2 vs. PFL : 20.0 months, $p=0.01$ ).

\section{DISCUSSION}

Various surgical methods have been developed for cervical spinal diseases. LF is one of the most recent procedures introduced for cervical disorders. It provides better long-term stability than laminectomy alone by inducing arthrodesis, and has a wider surgical indication than laminoplasty, allowing the reduction of kyphosis to lordosis ${ }^{1}$. However, postoperative neck pain, kyphosis, pseudoarthrosis, pseudomembrane formation are considered drawbacks for this procedure ${ }^{7,912)}$. We hypothesized that the posterior column can be preserved after LF by restoring the posterior column, including the laminae, at the very site where it had been cut. There are several merits for this procedure.

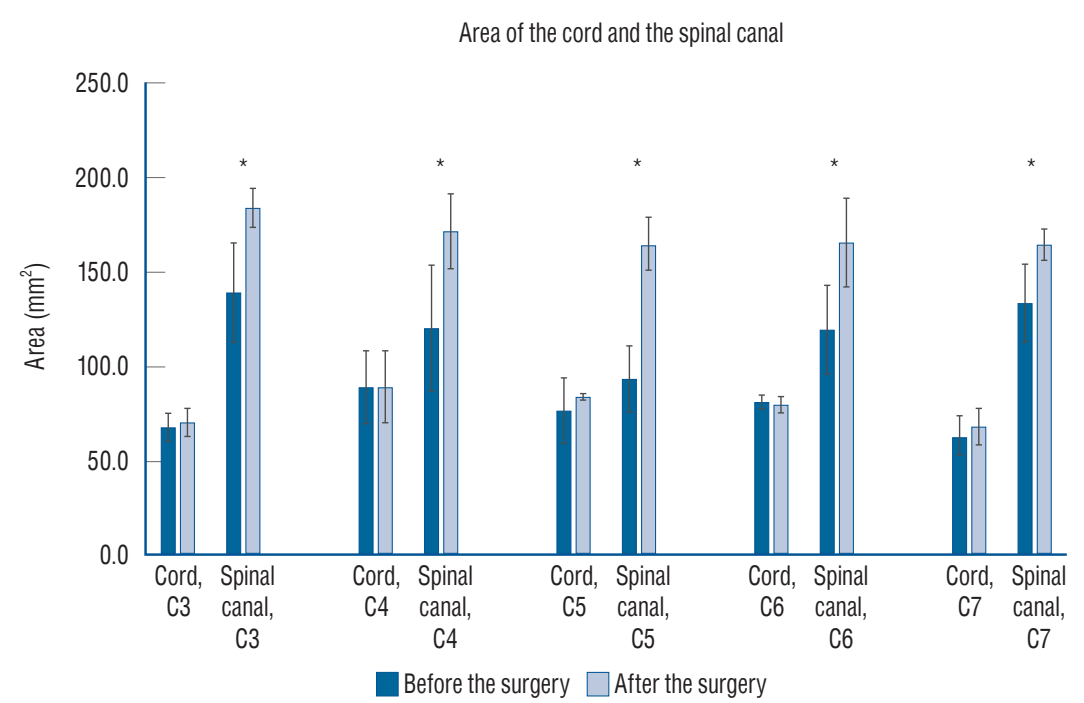

Fig. 7. The graph showing the area of the cord and the spinal canal from C3 to C7 which was measured at the T2-weighted axial magnetic resonance image. There were statistically significant differences in the area of the spinal canal from C3 to C7, between before and after the surgery (marked with stars). 
First, weight bearing for the anterior and middle columns can be distributed to the posterior column by bony reconstruction and muscle attachment, thereby preventing postoperative kyphotic change. Although the incidence of postoperative kyphosis is lower in LF than in laminectomy alone, postoperative kyphosis is a demanding complication after both procedures ${ }^{1,18)}$. In our study, the SL angle difference between the preoperative time and the last follow-up was $-0.3^{\circ}$ for conventional LF and $4.7^{\circ}$ for PFL ( $p=0.04$ ). The SL angle difference can be interpreted as the curvature maintenance capacity, which is related to postoperative kyphosis. By reattaching the posterior bony structures to their original sites, through the bone fusion of the floating lamina which was initially adhered to the paraspinal muscles, tendons, and upper and lower spinous processes, the progression of postoperative kyphosis can be mitigated. Since the posterior column dominantly supports the cervical load compared to the anterior vertebral body ${ }^{20,23)}$ and the large amount of laminectomy is the risk factor of the postoperative kyphosis ${ }^{6,16)}$, gutter bone fusion is believed to mandatory for the stabilization of the cervical alignment after the surgery.

Second, after bony structure reconstruction with gutter fusion, it is possible to physically protect the spinal cord from the back. Managing the empty space after laminectomy is an important but frequently overlooked issue. There was definite bony bridging at the gutter between the lateral mass and the lamina in our study in follow-up CT 1 year after the operation. Only after bone healing with gutter fusion did the complication risk at the empty space decrease.

Third, pseudomembrane formation can be prevented. Several reports have indicated that postlaminectomy pseudomembrane is a result of epidural hematoma or epidural scarring and a source of chronic inflammation and neurological deterioration ${ }^{12,24}$. After covering the bony cap of the spinal cord, pseudomembrane formation at the operation site is less likely.

Lastly, it is well known that dead space management is crucial for wounds. A major principle in managing the dead space is reducing the amount of space at the site through soft tissue reconstruction and bony support ${ }^{8}$. This is extremely impor-
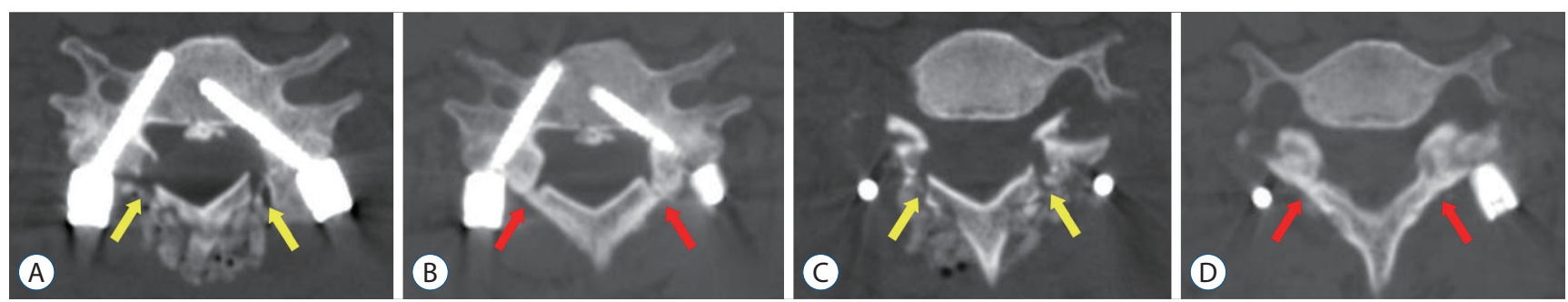

Fig. 8. Postoperative computed tomography (CT) images of a 67-year old man who received posterior floating laminotomy at C5-7. Follow-up CT 1 year after the operation revealed the formation of bony continuity at the gutter between the lateral mass and the lamina ( $B$ and $D)$, whereas immediate postoperative CT showed a gap between the lateral mass and the lamina ( $\mathrm{A}$ and $\mathrm{C}$ ). Red arrows indicate the formation of bony continuity at the gutter and yellow arrows indicate the gap between the lateral mass and the lamina.
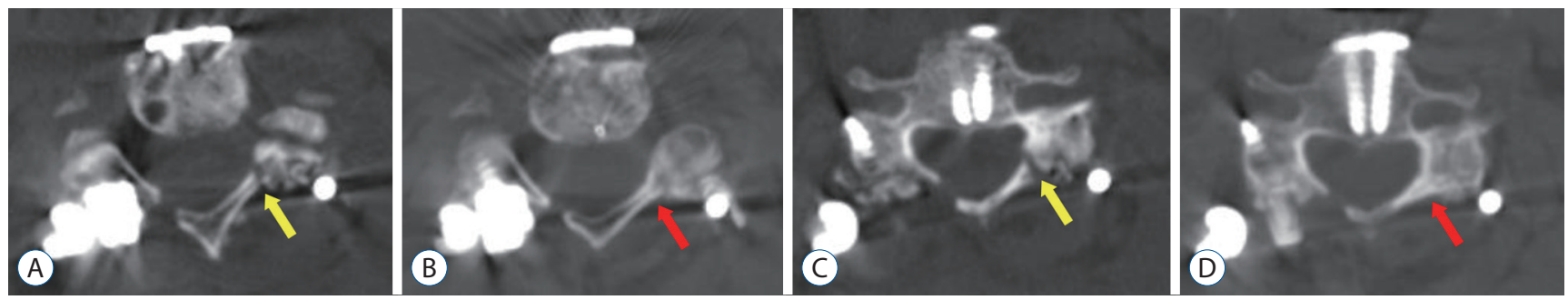

Fig. 9. Postoperative computed tomography (CT) images of an 80-year old woman who received posterior floating laminotomy (PFL) at C3-7. She had received anterior cervical discectomy and fusion and was diagnosed with an instrument malposition via postoperative X-ray. She underwent instrument removal and another PFL surgery. Follow-up CT 1 year after the operation revealed the formation of bony continuity at the gutter between the lateral mass and the lamina ( $B$ and D), whereas immediate postoperative CT showed a gap between the lateral mass and the lamina ( $A$ and $C$ ). Red arrows indicate the formation of bony continuity at the gutter and yellow arrows indicate the gap between the lateral mass and the lamina. 
Table 5. Perioperative outcomes for patients in conventional LF and PFL groups

\begin{tabular}{|c|c|c|c|}
\hline & $\begin{array}{c}\text { Conventional } \\
\text { LF }\end{array}$ & PFL & $p$-value \\
\hline Number of patients & 66 & 19 & \\
\hline $\begin{array}{l}\text { Number of levels fused } \\
\text { (levels) }\end{array}$ & $2.8 \pm 1.2$ & $3.0 \pm 1.4$ & 0.48 \\
\hline Screw fixation method & & & 0.42 \\
\hline CPS & $424(87.4)$ & $131(90.3)$ & \\
\hline LMS & $61(12.6)$ & $14(9.7)$ & \\
\hline $\begin{array}{l}\text { Total operation time } \\
\text { (minutes) }\end{array}$ & $169.6 \pm 60.3$ & $223.7 \pm 65.9$ & 0.01 \\
\hline $\begin{array}{l}\text { Operation time/fusion level } \\
\text { (minutes/level) }\end{array}$ & $72.3 \pm 35.8$ & $90.2 \pm 46.9$ & 0.08 \\
\hline Estimated blood loss (mL) & $270.2 \pm 91.1$ & $294.7 \pm 62.5$ & 0.28 \\
\hline $\begin{array}{l}\text { Length of hospital stay } \\
\text { (days) }\end{array}$ & $23.3 \pm 17.5$ & $23.3 \pm 27.1$ & 0.99 \\
\hline Non-pathologic fever & $15(22.7)$ & $2(10.5)$ & 0.34 \\
\hline \multicolumn{4}{|l|}{$\begin{array}{l}\text { Perioperative } \\
\text { complications }\end{array}$} \\
\hline Wound infection & $5(7.6)$ & $0(0.0)$ & 0.58 \\
\hline Seroma & $6(9.1)$ & $1(5.3)$ & 1.00 \\
\hline C5 palsy & $1(1.5)$ & $1(5.3)$ & 0.39 \\
\hline Screw fracture & $1(1.5)$ & $0(0.0)$ & 1.00 \\
\hline Restenosis due to trauma & $0(0.0)$ & $1(5.3)$ & 0.22 \\
\hline Follow-up (months) & $35.2 \pm 20.8$ & $20.0 \pm 9.9$ & 0.01 \\
\hline
\end{tabular}

Values are presented as mean \pm standard deviation or number (\%). LF : laminectomy and fusion, PFL : posterior floating laminotomy, CPS : cervical pedicle screw, LMS : lateral mass screw

tant not only for traumatic injuries, but also for infectious diseases ${ }^{5,17)}$. Dead space at the surgical site is considered to be related to hematoma or seroma formation, which increases the risk of bacterial growth ${ }^{14)}$. Several methods, including suction drain, vacuum-assisted wound closure, myocutaneous flap, and antibiotic loaded bone cement, have been proved to reduce the postoperative dead space ${ }^{14,19)}$. Postoperative seroma is also known to occur in cervical spine surgery ${ }^{27,29)}$. Wound infection and seroma occurred less frequently in the PFL group than in the conventional LF group but no statistical significance was found in our study due to the small patient number of the PFL group. This result may be attributed to the greater decrease in dead space after the operation in the PFL group than in the conventional LF group due to the restoration of bony structures.
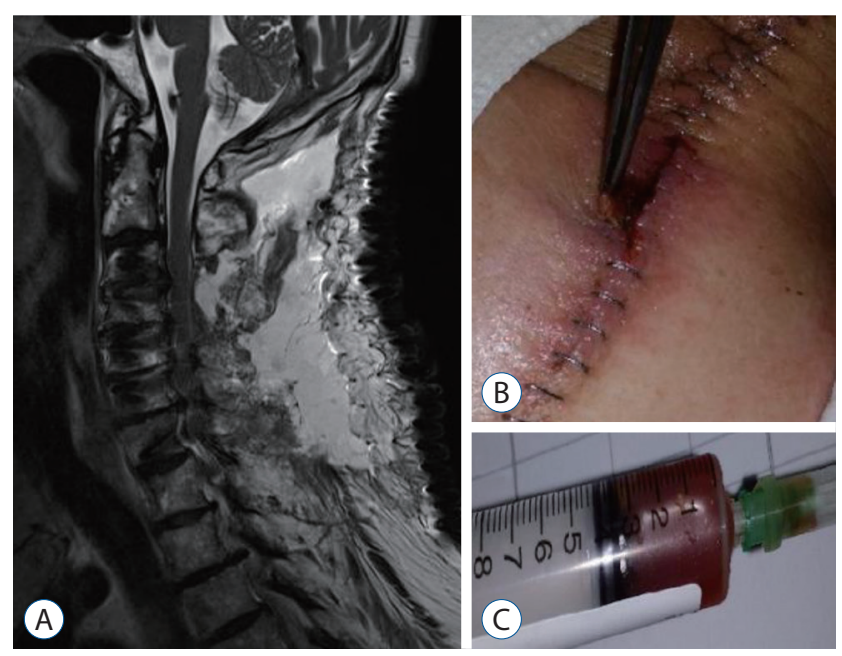

Fig. 10. Postoperative seroma is demonstrated. A : Postoperative magnetic resonance image 12 days after conventional laminectomy and fusion (A) showed T2 hyperintense fluid collection overlaying the C2-7 levels consistent with a postoperative seroma. B : The superficial wound was clear except for some pinkish margins at the middle of the wound due to the pressure of the seroma. $C$ : The aspirated seroma in revision surgery was a clear and reddish fluid, which did not reveal any bacterial growth.

It is important to ensure that the re-attached laminae are completely fused with the adjacent bone. Restenosis due to postoperative cervical trauma occurred in one patient in the PFL group. This patient slipped down at the stairway in a month after the PFL and came to the clinic with arm and leg weakness. CT revealed that the restored laminae compressed the spinal cord. Emergent operation was performed, and the patient recovered to the previous normal neurologic status. This case highlights the significance of the bone fusion achievement after surgery since the bone fusion is essential not only for the stabilizing the alignment but also for the prevention of restenosis, especially after the PFL surgery. It is generally believed that it takes at least 6 months for complete bone fusion ${ }^{21)}$. Therefore, to obtain satisfactory results after surgery, it is necessary to be careful during the postoperative follow-up period.

Our study does have several limitations. First, since this study is a retrospective comparative study, randomized distribution to the conventional LF group and the PFL group was not possible. Second, the number of patients in the PFL group was relatively small compared to the number in the conventional LF group. Third, the follow-up period for the PFL group was shorter than that for the conventional LF group and was 
short overall. Long term follow-up for both groups is needed. PFL surgery has only been performed for a short period at our institute. In the next report, it will be possible to compare the two groups with similar follow-up and number of patients.

\section{CONCLUSION}

PFL was found to be safe and to achieve good results for cervical disorders. Our study showed that PFL achieves excellent alignment preservation as well as lower complication rates compared to conventional LF. Additionally, PFL demonstrated satisfactory improvements in several functional outcomes, specifically mJOA, NDI, VAS-N, and VAS-A scores. However, because of the aforementioned limitations, we could not draw a strong conclusion that PFL is superior to conventional LF. More number of the PFL patients are needed to make strong conclusions. Further randomized controlled trials comparing the conventional LF and PFL for cervical diseases should be performed to reach a more obvious conclusion.

\section{CONFLICTS OF INTEREST}

No potential conflict of interest relevant to this article was reported.

\section{INFORMED CONSENT}

This type of study does not require informed consent.

\section{AUTHOR CONTRIBUTIONS}

\author{
Conceptualization : JHP \\ Data curation : JHP \\ Formal analysis : HKS \\ Methodology : JHP \\ Project administration : JHP \\ Visualization : HKS \\ Writing - original draft : HKS \\ Writing - review \& editing : JHP
}

\section{ORCID}

Hong Kyung Shin https://orcid.org/0000-0001-8182-3321

Jin Hoon Park https://orcid.org/0000-0002-0903-3146

\section{References}

1. Anderson PA, Matz PG, Groff MW, Heary RF, Holly LT, Kaiser MG, et al. : Laminectomy and fusion for the treatment of cervical degenerative myelopathy. J Neurosurg Spine 11 : 150-156, 2009

2. Baba H, Furusawa N, Imura S, Kawahara N, Tsuchiya H, Tomita K : Late radiographic findings after anterior cervical fusion for spondylotic myeloradiculopathy. Spine (Phila Pa 1976) 18 : 2167-2173, 1993

3. Baisden J, Voo LM, Cusick JF, Pintar FA, Yoganandan N : Evaluation of cervical laminectomy and laminoplasty. A longitudinal study in the goat model. Spine (Phila Pa 1976) 24 : 1283-1288; discussion 1288-1289, 1999

4. Blizzard DJ, Caputo AM, Sheets CZ, Klement MR, Michael KW, Isaacs $\mathrm{RE}$, et al. : Laminoplasty versus laminectomy with fusion for the treatment of spondylotic cervical myelopathy: short-term follow-up. Eur Spine J $26: 85-93,2017$

5. Cierny $G$ 3rd : Surgical treatment of osteomyelitis. Plast Reconstr Surg 127 Suppl 1 : 190S-204S, 2011

6. Deutsch H, Haid RW, Rodts GE, Mummaneni PV : Postlaminectomy cervical deformity. Neurosurg Focus 15 : E5, 2003

7. Fehlings MG, Santaguida C, Tetreault L, Arnold P, Barbagallo G, Defino $H$, et al. : Laminectomy and fusion versus laminoplasty for the treatment of degenerative cervical myelopathy: results from the AOSpine North America and International prospective multicenter studies. Spine J 17 : 102-108, 2017

8. Gage MJ, Yoon RS, Gaines RJ, Dunbar RP, Egol KA, Liporace FA : Dead space management after orthopaedic trauma: tips, tricks, and pitfalls. J Orthop Trauma 30 : 64-70, 2016

9. Highsmith JM, Dhall SS, Haid RW Jr, Rodts GE Jr, Mummaneni PV : Treatment of cervical stenotic myelopathy: a cost and outcome comparison of laminoplasty versus laminectomy and lateral mass fusion. J Neurosurg Spine $14: 619-625,2011$

10. Hukuda S, Mochizuki T, Ogata M, Shichikawa K, Shimomura Y : Operations for cervical spondylotic myelopathy. A comparison of the results of anterior and posterior procedures. J Bone Joint Surg Br 67 : 609-615, 1985

11. Kaptain GJ, Simmons NE, Replogle RE, Pobereskin L : Incidence and outcome of kyphotic deformity following laminectomy for cervical spondylotic myelopathy. J Neurosurg 93(2 Suppl) : 199-204, 2000

12. Kitahara T, Hanakita J, Takahashi T : Postlaminectomy membrane with dynamic spinal cord compression disclosed with computed tomographic myelography: a case report and literature review. Spinal Cord Ser Cases $3:$ 17056, 2017

13. Lawrence BD, Jacobs WB, Norvell DC, Hermsmeyer JT, Chapman JR, 
Brodke DS : Anterior versus posterior approach for treatment of cervical spondylotic myelopathy: a systematic review. Spine (Phila Pa 1976) 38(22 Suppl 1) : S173-S182, 2013

14. Masuda S, Fujibayashi S, Otsuki B, Kimura H, Matsuda S : Efficacy of target drug delivery and dead space reduction using antibiotic-loaded bone cement for the treatment of complex spinal infection. Clin Spine Surg 30 : E1246-E1250, 2017

15. McCormack HM, Horne DJ, Sheather S: Clinical applications of visual analogue scales: a critical review. Psychol Med 18 : 1007-1019, 1988

16. McLaughlin MR, Wahlig JB, Pollack IF : Incidence of postlaminectomy kyphosis after Chiari decompression. Spine (Phila Pa 1976) 22 : 613617, 1997

17. Metsemakers WJ, Fragomen AT, Moriarty TF, Morgenstern M, Egol KA, Zalavras $C$, et al. : Evidence-based recommendations for local antimicrobial strategies and dead space management in fracture-related infection. J Orthop Trauma 34 : 18-29, 2020

18. Miyazaki K, Tada K, Matsuda Y, Okuno M, Yasuda T, Murakami H : Posterior extensive simultaneous multisegment decompression with posterolateral fusion for cervical myelopathy with cervical instability and kyphotic and/or S-shaped deformities. Spine (Phila Pa 1976) 14 : 1160-1170, 1989

19. Ogura K, Miyamoto S, Sakuraba M, Chuman H, Fujiwara T, Kawai A : Immediate soft-tissue reconstruction using a rectus abdominis myocutaneous flap following wide resection of malignant bone tumours of the pelvis. Bone Joint J 96-B : 270-273, 2014

20. Pal GP, Sherk HH : The vertical stability of the cervical spine. Spine (Phila
Pa 1976) $13:$ 447-449, 1988

21. Pilitsis JG, Lucas DR, Rengachary SS : Bone healing and spinal fusion. Neurosurg Focus 13 : e1, 2002

22. Prasad SS, O'Malley M, Caplan M, Shackleford IM, Pydisetty RK : MRI measurements of the cervical spine and their correlation to Pavlov's ratio. Spine (Phila Pa 1976) 28 : 1263-1268, 2003

23. Raynor RB, Pugh J, Shapiro I: Cervical facetectomy and its effect on spine strength. J Neurosurg $63: 278-282,1985$

24. Rönnberg K, Lind B, Zoega B, Gadeholt-Göthlin G, Halldin K, Gellerstedt $M$, et al. : Peridural scar and its relation to clinical outcome: a randomised study on surgically treated lumbar disc herniation patients. Eur Spine J 17 : 1714-1720, 2008

25. Rüegg TB, Wicki AG, Aebli N, Wisianowsky C, Krebs I : The diagnostic value of magnetic resonance imaging measurements for assessing cervical spinal canal stenosis. J Neurosurg Spine 22 : 230-236, 2015

26. Seo J, Park JH, Song EH, Lee YS, Jung SK, Jeon SR, et al. : Postoperative nonpathologic fever after spinal surgery: incidence and risk factor analysis. World Neurosurg $103:$ 78-83, 2017

27. Tan LA, Kasliwal MK, Traynelis VC : Surgical seroma. J Neurosurg Spine 19 : 793-794, 2013

28. Vernon $\mathrm{H}$, Mior $\mathrm{S}$ : The neck disability index: a study of reliability and validity. J Manipulative Physiol Ther 14 : 409-415, 1991

29. Yew A, Kimball J, Lu DC : Surgical seroma formation following posterior cervical laminectomy and fusion without rhBMP-2: case report. J Neurosurg Spine $19: 297-300,2013$ 